\title{
Usos de TIC y software especializado en la investigación cualitativa. Un panorama
}

\author{
María Elena Giraldo Ramírez* \\ Gloria M. Álvarez Cadavid* \\ Clemencia del Pilar Navarro Plazas**
}

Artículo recibido:

6 de agosto de 2019

Artículo aceptado:

29 de octubre de 2019

Artículo de revisión

\section{Resumen}

Este artículo forma parte de la investigación "Usos y apropiación de TIC en las prácticas investigativas cualitativas y en los procesos de formación investigativa en educación superior". Lo que aquí se presenta es el resultado de la primera fase referente a la revisión de la literatura sobre el tema, en los aspectos epistemológicos, metodológicos y prácticos de la mediación tecnológica en la investigación cualitativa. El objetivo de este artículo es exponer los hallazgos encontrados en la literatura acerca de las discusiones, perspectivas y experiencias sobre los usos de TIC y de software especializado tipo CAQDAS en las prácticas investigativas cualitativas.

* Escuela de Educación y Pedagogía, Universidad Pontificia Bolivariana, Colombia maria.giraldo@upb.edu.co_gloria.alvarez@upb.edu.co ** Software Shop de Colombia_clemencia@software-shop.com INVESTIGACIÓN BIBLIOTECOLÓGICA, vol.34, núm. 84, julio/septiembre, 2020, México, ISSN: 2448-8321 pp. 33-57 
El análisis realizado parte de una caracterización de la producción bibliográfica recopilada, en donde se analizan aspectos como la frecuencia de publicación, los autores, idiomas, temáticas, entre otros asuntos. Los hallazgos más significativos revelan poca referencia al uso de TIC en las fases iniciales de los procesos investigativos, siendo los más recurrentes los gestores bibliográficos. Las herramientas tipo CAQDAS se siguen usando, fundamentalmente, en fases de análisis de datos; son pocos los usos específicos, identificados para actividades como la revisión de literatura conducente a construcción de estados del arte, o enfocados a apoyar el diseño de la investigación.

Palabras clave: Apropiación Tecnológica; CAQDAS; Investigación Cualitativa; Tecnologías de la Información y la Comunicación (TIC)

Uses of ICT and software specialized in qualitative research. A perspective

María Elena Giraldo Ramírez, Gloria M. Álvarez Cadavid and Clemencia del Pilar Navarro Plazas

\section{Abstract}

This article is part of the research ICT uses and appropriation in qualitative research practices and formative research processes in higher education. What is here presented is the result of the first stage of the process, which refers to the reviewing of literature on the theme: the epistemological, methodological and practical aspects of the technological mediation in qualitative research. The aim of this is to expose what was founding the literature about perspectives, discussions and experiences about the uses of ICT and the specialized software type CAQDAS in qualitative research practices.

The analysis is part of a characterization of the compiled bibliographic production, which focus aspects such as frequency of publication, authors, languages, themes and other matters. The more significative findings reveal poor reference to the use of ICT in the initial phases of research processes, being the most recurred to the bibliographical administrators. Tools type CAQDAS are still used, above all for the analysis of data phases; specific uses are a few and they are a 
few and they are identified for activities as revision of literature leading to construction of states of the art, or focused to support the design of the research.

Keywords: Technological Appropriation; CAQDAS; Qualitative Research; Information And Communication Technologies (ICT)

\section{INTRODUCCIÓN}

T as discusiones sobre las ventajas o desventajas del uso de software especializado para el análisis cualitativo de datos, tipo CAQDAS (Computer Assisted Qualitative Data Analysis), parecen estar a la orden del día. De hecho, y de acuerdo con la exhaustiva revisión de Cisneros Puebla (2003: 289), “[... ] existe ya un corpus de materiales que permiten pensar al Análisis Cualitativo Asistido por Computadora (ACAC) como un campo singular y privilegiado del conocimiento". Caso contrario sucede cuando hablamos de la aplicación del software especializado a otras fases de la investigación cualitativa, a las cuales se hace poca referencia en la literatura (Fielding, Lee y Mangabeira, 2004; Fielding y Lee, 1996 y Valles, 2005).

Lo anterior, sumado al uso de herramientas digitales en la investigación cualitativa, que aquí se asumen en lo que se denominan tecnologías de la información y la comunicación (TIC), constituyen los dos horizontes de interés en este artículo: los usos de software especializado para el análisis cualitativo de datos así como de las herramientas digitales TIC en todo el proceso investigativo, no exclusivamente en la etapa del análisis de datos. Lo cual se hace insoslayable para lograr una comprensión más clara de la función de estas herramientas dentro de los procesos de investigación cualitativa.

Este fue un punto de partida para la hipótesis de trabajo, en relación con las prácticas investigativas de tipo cualitativo mediadas tecnológicamente: el incremento de TIC plantea sólo una diversificación en las prácticas de usos en la investigación o va más allá y configura un nuevo enfoque teórico y metodológico en los procesos de apropiación de estas tecnologías en la investigación cualitativa (Álvarez, Giraldo y Navarro, 2017).

De acuerdo con varios autores (Arriazu Muñoz, 2007; Bourdon, 2002; Cisneros Puebla, 2003, 2011; Cisneros Puebla y Davidson, 2012; MacMillan y Koenig, 2004; Roberts y Wilson, 2002; Sin, 2008 y Valles, 2001) hay una serie de decisiones pragmáticas derivadas de la necesidad de gestionar tanto la información como la complejidad logística de la investigación que pueden 
afectar la forma en que los datos se entienden y son interrogados, lo cual, inevitablemente, influye en la naturaleza del proceso investigativo.

Estos hallazgos se presentan en cuatro partes. En la primera se inicia con un recorrido de los momentos en que surgen ciertos hitos tecnológicos y las prácticas que con ellos se inauguran; la segunda describe la metodología y el corpus de datos analizado; la tercera presenta los resultados a partir de las búsquedas realizadas en las distintas bases de datos y fuentes académicas; y la cuarta plantea las conclusiones desde la relación entre el repertorio de usos, las herramientas utilizadas y las fases de la investigación en las que predominan dichas prácticas.

Hitos de LA EVOLUCión DE SOFTWARE ESPECIALIZADO Y DE TIC EN LOS PROCESOS DE LA INVESTIGACIÓN CUALITATIVA

Para poder comprender este comportamiento particular de la producción bibliográfica, relacionada con usos de software especializado tipo CAQDAS y de TIC en investigación, es importante tener en cuenta el mencionado predominio hasta la década de los ochenta de la tradición manual en el análisis de datos cualitativos y el desarrollo histórico de la incorporación de uso de TIC a los procesos de investigación en general. Para ello, se optó por identificar algunos de los principales hitos tecnológicos que sirven de marco de referencia y contexto histórico de los usos de CAQDAS y de TIC en la investigación cualitativa, mostrados a través de una línea de tiempo (Figura 1).

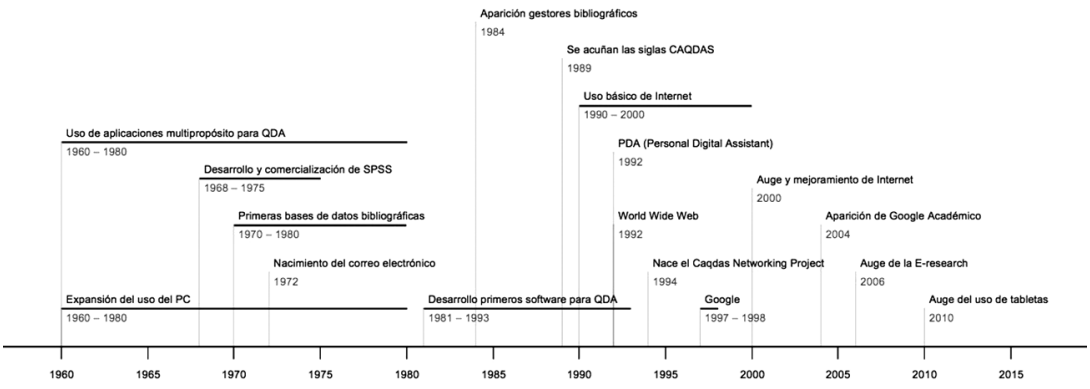

Figura 1. Hitos de la evolución de usos de software especializado y de TIC en los procesos de investigación cualitativa 
La década de 1960. Aunque el desarrollo de las computadoras se remonta a los años cuarenta, sólo hasta los sesenta comienza una verdadera expansión de su uso en instituciones y hogares. Dicho cambio representa un paso del uso restringido para científicos especializados a uno más generalizado por parte de públicos de diversas áreas. Un hito representativo en esta década es el desarrollo y comercialización del SPSS (Statistical Package for the Social Sciences), uno de los software más conocidos para análisis cuantitativo en las ciencias sociales. A la par, algunos procesos de análisis de datos cualitativos (en inglés Qualitative Data Analysis - QDA) se llevan a cabo en aplicaciones multipropósito, es decir, aquellas no especializadas, la mayoría de ellas asociadas a procesadores de texto.

La década de 1970. Dos eventos marcan estos años, el primero de ellos es el surgimiento de las bases de datos bibliográficas que le dan otro nivel a la gestión del conocimiento, y el segundo, el surgimiento del correo electrónico pues, aunque hubo versiones anteriores de comunicación entre equipos, como Mailbox en 1965, el invento del correo electrónico como lo conocemos se le atribuye a Ray Tomlinson en 1972 (Peter, 2010).

La década de 1980. Estos años son señalados como el punto de ruptura en el uso de software especializado para apoyo del análisis cualitativo (Chernobilsky, 2006; Cisneros Puebla, 2003 y Trejo Teruel, 2011). Surgen entonces las primeras versiones comerciales de CAQDAS: Nud*ist, 1981; Ethnograph, 1984; ATLAS.ti., 1993. Igualmente, aparecen los primeros software de gestión bibliográfica como Reference Manager y más tarde EndNote (aunque ya en 1973 había sido desarrollado ISIS, para gestionar información zoológica). Y para completar la relevancia de esta década, en 1989 Nigel Fielding y Ray Lee (1996) acuñan el término Computer Assisted Qualitative Data Analysis (CAQDAS).

La década de 1990. Tres hitos de grandes repercusiones metodológicas y epistemológicas marcan esta década: el primero fue el lanzamiento de la World Wide Web (WWW) o red informática mundial (Fielding et al., 1999), aunque su creador Tim Berners-Lee ya trabajaba en ella desde finales de los años ochenta. El segundo hito, propiciado por el anterior, fue la configuración de internet como escenario de investigación dada su rápida popularización; y el tercer hito fue el surgimiento del Personal Digital Assistant (PDA), dispositivo de pantalla sensible al tacto, portátil, con conectividad a redes vía módem y reconocimiento de escritura, precursor de los actuales dispositivos móviles que con sus prestaciones han modificado las rutinas de comunicación contemporáneas.

Adicionalmente, en esta década es importante mencionar dos proyectos también de gran impacto: el primero de ellos Google, con repercusiones 
a escala global y considerado el mejor motor de búsqueda de la historia; el segundo, con un marco de interés muy específico, se trata del CAQDAS Networking Proyect, liderado por varios investigadores ingleses y que da un espaldarazo académico al uso de CAQDAS en investigación social.

La década del 2000. Se puede afirmar que esta década se caracteriza por la consolidación y el mejoramiento de algunos de los hitos mencionados anteriormente. Por ejemplo, internet como escenario de investigación abre un espacio para ampliar los objetos de estudio de la sociología y la antropología, evidencia de ello es el trabajo pionero de Christine Hine (2000) con las reflexiones sobre la etnografía virtual. En forma similar, se observa un desarrollo exponencial de dispositivos móviles con la entrada de las tabletas y las aplicaciones que lo acompañan, muchas de ellas con funciones para la investigación tanto en la captura de datos (registro de audio y video) como en actividades más especializadas (por ejemplo, el APP de ATLAS.ti para tabletas).

La década de 2010. En este periodo se asiste a la refinación tecnológica de los dispositivos móviles que han incrementado las posibilidades del trabajo colaborativo en tiempo real, la ampliación de la interacción multiplataforma y el aumento del uso de redes sociales en la investigación, ya no sólo como objeto de estudio sino como herramienta de recolección de información. La portabilidad y el acceso libre a aplicaciones y dispositivos han hecho que el registro y manejo de los datos, en general, sea muy ágil y flexible.

Finalmente, y luego del anterior recorrido cronológico, es importante resaltar, como lo indica Cisneros Puebla (2003), que la aparición de un desarrollo tecnológico o una herramienta especializada no es necesariamente un indicador de la expansión de su uso, más aún en Iberoamérica, en donde las restricciones de costos, idioma, acceso, competencias tecnológicas, divulgación y capacitación pueden ser factores determinantes para el uso o no de algunas de estas herramientas y sus posibilidades. Así, se procede a continuación, desde el corpus específico, a aportar evidencias sobre la producción bibliográfica respecto al tema para resaltar de qué manera se construye el conocimiento sobre este campo tan particular de la apropiación tecnológica.

\section{Metodología}

\section{Producción académica y científica sobre usos de TIC en investigación cualitativa}

El análisis aquí presentado se plantea como una caracterización de la producción bibliográfica recopilada a lo largo del proceso de investigación sobre 
usos de TIC en investigación cualitativa (De Bellis, 2009; Jiménez-Contreras, 2000). Se analizan aspectos como la frecuencia de publicación, los autores, los idiomas, las temáticas, entre otros, como datos relevantes para comprender no sólo las características del uso de software reportado en esta producción académica, sino también las discusiones generadas acerca del uso y no uso de estas herramientas, y el tipo de información con la que cuentan los investigadores que se interesan en el tema (Cisneros Puebla et al., 2006; Cisneros Puebla et al., 2009).

En este rastreo se encontraron cuatro trabajos de revisión similares al que aquí se presenta. El primero, de MacMillan y Koenig (2004: 179-180), hace un análisis de las publicaciones de la base de datos Sociological Abstracts; encuentra una diferencia importante entre los artículos de enfoque cualitativo y cuantitativo relacionados con el uso de herramientas de software en procesos de investigación. Mientras que las referencias al uso de software para análisis cualitativo se limitaban a 31 artículos, encontraron 220 artículos referidos al uso de software para análisis estadístico. Igualmente, reportan cómo 24 de estos 31 artículos hacen referencia a CAQDAS y, de éstos, 23 se centraban en discutir las ventajas y desventajas del software, lo que no ocurría en el caso de los artículos de enfoque cuantitativo, en donde el tema central de la publicación era la investigación misma. Cabe anotar que, de estos últimos, 10 habían sido escritos por los mismos desarrolladores de software.

El segundo trabajo, de Graham Gibbs (2013), realiza una búsqueda similar sobre uso de CAQDAS en análisis cualitativo en revistas de ciencias sociales, encontrando artículos desde 1983 hasta 2011. Sin embargo, de acuerdo con los resultados obtenidos, señala que esta producción sólo comienza a reportar un crecimiento importante a partir del 2000 (menos de 100 artículos en 2000, 200 artículos en 2005 y 400 en 2011), mientras que antes de esta fecha solamente aparecen unos pocos artículos aislados por año.

El tercer estudio de revisión, de Woods, Macklin y Lewis (2016), se centra en el impacto del uso de CAQDAS en la reflexividad del investigador a través de la revisión de tres décadas de literatura (1980-2011). Este estudio pretende entender cómo el uso de CAQDAS puede influir en las prácticas de los investigadores y en los resultados de la investigación, lo cual plantea importantes consideraciones metodológicas que, en últimas, contribuyen a mejorar la reflexividad del investigador.

En el cuarto estudio, Paulus et al. (2017) realizan un análisis del discurso de 763 artículos publicados entre 1994-2013, particularmente en el área de Ciencias de la Salud, sobre el lenguaje utilizado por los investigadores al informar sobre el uso de software de análisis cualitativo de datos (Qualitative Data Analysis Software - Q-DAS). Este estudio revela cómo la mayoría de 
los investigadores proporcionan pocos detalles del uso de Q-DAS, más allá de nombrarlos como apoyo al análisis de datos, lo cual, concluyen los investigadores, incide en la poca transparencia de los procesos de investigación y contribuye a que persistan conceptos erróneos sobre el papel y el impacto de los Q-DAS en la investigación cualitativa.

\section{Corpus documental}

Se realizó una búsqueda con corte a enero de 2019 en bases de datos y fuentes académicas, y en artículos relacionados y citados en la literatura sobre el tema. Se utilizaron descriptores en español e inglés y distintas combinaciones de los mismos: qualitative research, e-research, research computing, e-social science, technology, Computer Assisted Analysis, QDA, CAQDAS, software computing, ICT use, research process, data analysis, uso de TIC, investigación cualitativa, tecnologías, proceso de investigación, análisis de datos. La captura de los datos se realizó con el apoyo de software para gestión bibliográfica (Zotero y EndNote).

Luego, se realizó una selección en la que se tuvieron en cuenta aspectos como la pertinencia para el tema, la fuente, la disponibilidad del documento y la revisión inicial del contenido a partir del título, abstract y cuerpo de la publicación. Con esta selección se procedió a etiquetar nuevos campos requeridos para la caracterización como el idioma, categorías temáticas y herramientas de TIC asociadas a fases específicas de la investigación. De una base inicial de 348 artículos fueron seleccionados 244 de acuerdo con los criterios mencionados anteriormente. No se acordó una restricción específica con respecto al año de publicación ya que el objetivo era poder rastrear algunos patrones de frecuencia de publicación en esta selección.

\section{Resultados}

\section{Distribución por año de publicación}

Los artículos abarcan un rango de poco más de 20 años comprendidos entre 1993 y 2019. Se evidencia un lapso de tiempo entre el surgimiento de los primeros software especializados en los años ochenta (Chernobilsky, 2006; Cisneros Puebla, 2003 y Trejo Teruel, 2011) y la divulgación académica de los mismos. Esto podría estar asociado a varios asuntos: uno, el ritmo pausado de los procesos de divulgación académica y científica de la época; dos, la 
lenta organización de la comercialización de software de pago; tres, el hecho de que la adquisición del software, por lo general, estaba soportada en medios de grabación físicos (disquete, $\mathrm{CD}$ ); cuatro, el uso de computadoras personales no estaba aún tan extendido. Igualmente, en concordancia con los hallazgos de Gibbs (2013), se observa un comportamiento más dinámico en el crecimiento del número de artículos encontrados a partir de 2002, fecha desde la cual la discusión del tema se ha mantenido vigente.

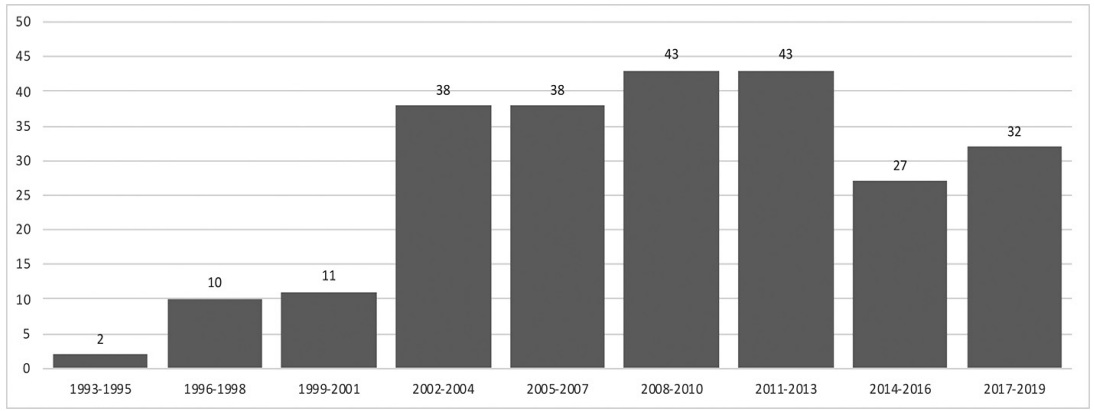

Figura 2. Distribución de artículos académicos por año de publicación: 1993-2019

\section{Idioma de publicación}

Este aspecto resulta ser un factor determinante en el acceso no sólo al conocimiento de las herramientas de software sino también a las discusiones y reflexiones epistemológicas y metodológicas sobre su uso. Si bien los términos de búsqueda se usaron en inglés y en español, al analizar el idioma de publicación de los artículos seleccionados para esta caracterización se encontró que $69.55 \%$ estaban escritos en idioma inglés, $27.98 \%$ en español y $2.47 \%$ en otros idiomas (alemán y portugués).

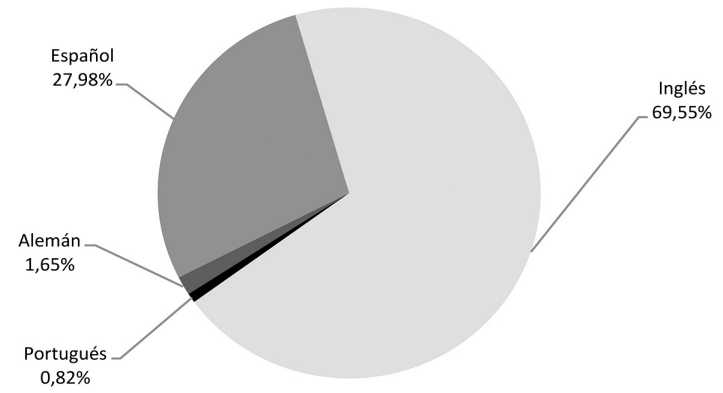

Figura 3. Distribución de artículos académicos por idioma 


\section{Tipo de herramientas mencionadas}

Para este análisis se tuvieron en cuenta las herramientas mencionadas en los artículos revisados (244), tanto en el título como en el abstract y en el cuerpo del texto. El criterio para obtener la frecuencia no fue el conteo de palabras sino la mención de las herramientas por artículo; por tanto, si en un artículo aparecía una misma herramienta mencionada varias veces, sólo se tuvo en cuenta una vez. A partir de este procedimiento se obtuvo una lista de herramientas utilizadas en la investigación cualitativa, las cuales fueron agrupadas por tipo (Figura 4).

Es importante hacer notar tres asuntos: primero, la herramienta más mencionada se refiere al uso de software tipo CAQDAS. Dado los descriptores priorizados, ello no representa una novedad; sin embargo, permite confirmar una tendencia ya consolidada de uso. Segundo, las menciones al uso de herramientas para tramitar datos en formatos de audio y video están en aumento, y tercero, algunas herramientas mencionadas aluden a software estadístico. Esto se presenta, sobre todo, en artículos que plantean métodos mixtos de investigación y de análisis asociados, por ejemplo, la sociolingüística cuantitativa o formas de analizar cuantitativamente datos cualitativos.

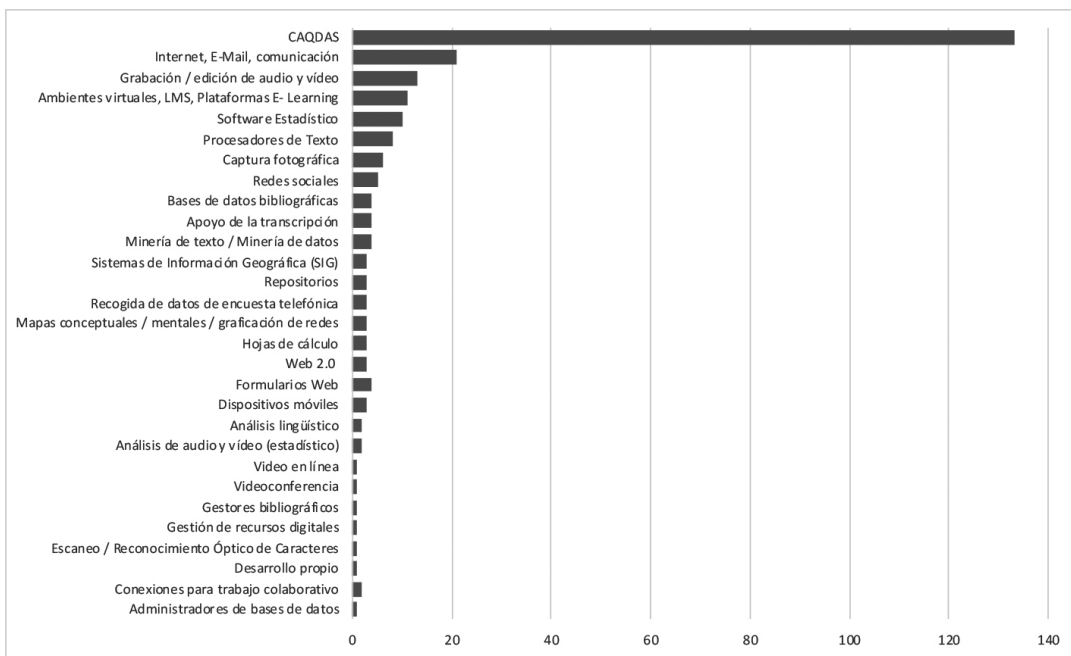

Figura 4. Tipo de herramientas por frecuencia en que aparecen mencionadas en los artículos revisados 


\section{Enfoque temático}

Para este tópico se siguió el mismo criterio que en el caso anterior, esto es, se realizó una clasificación temática de los artículos basada en el análisis de títulos, abstract y cuerpo de la publicación. Se obtuvo la frecuencia no sólo del tipo de herramientas TIC mencionadas sino del enfoque temático del artículo, categorizada de acuerdo con los usos de estas herramientas y las discusiones que suscitan. Este análisis permitió identificar recurrencias en cinco enfoques temáticos, a saber: Casos de uso, Debate sobre uso de TIC, Discusiones metodológicas, Experiencias de formación, y Comparación de herramientas.

Como se puede ver en el apartado anterior (Figura 4), la mayor parte de artículos se centran en CAQDAS (133), por lo que se estableció la relación entre el enfoque temático y el tipo de herramientas, agrupando las que no hacen referencia directa a CAQDAS en Otras herramientas (Figura 5). El enfoque predominante para CAQDAS es Casos de uso, donde los artículos se centran en cómo usar las herramientas, particularmente CAQDAS, para el análisis textual, el análisis de contenido, el análisis de redes sociales o para el análisis multimedia, seguido de Discusiones metodológicas, que agrupa categorías como el uso de herramientas TIC en enfoques mixtos de investigación, el rigor y la calidad en la investigación cualitativa, y las implicaciones metodológicas del uso de TIC. Para Otras herramientas, por el contrario, predomina Discusiones metodológicas y sigue Casos de uso.

En relación con Debate sobre uso de TIC, se identificaron los artículos cuyo foco era la discusión sobre las ventajas y desventajas del uso de TIC en la investigación cualitativa. Es importante señalar que, en muchos casos, estas discusiones tocaban necesariamente el tema metodológico, por lo que también se tomaron en cuenta en el anterior enfoque sobre las discusiones metodológicas. Por último, están las Experiencias de formación, centradas generalmente en la enseñanza de un software determinado, tipo CAQDAS, a estudiantes de posgrado, y Comparación de herramientas, que incluye los artículos que se dedican a comparar las funcionalidades y diferencias entre éstas, también muy centradas en CAQDAS, particularmente NVivo, ATLAS. ti, MaxQDA y, en menor medida, Transana y WebQDA. 


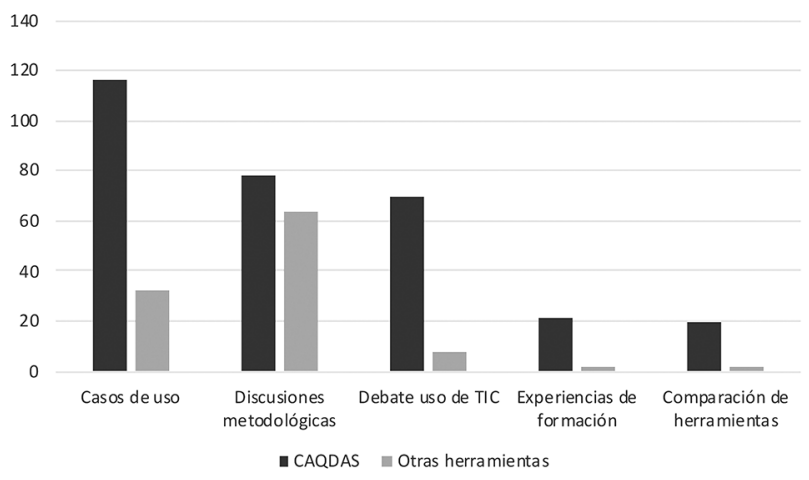

Figura 5. Frecuencia de artículos por tipo de herramienta y enfoque temático

Estos resultados evidencian un incremento en el interés por las implicaciones metodológicas del uso de TIC en la investigación cualitativa, a diferencia de los hallazgos de MacMillan y Koenig (2004), citados anteriormente. Se podría inferir que, tal vez, se esté superando el proceso de adaptación a la novedad que implica integrar el software al proceso investigativo cualitativo, característico de las primeras décadas, y se esté asistiendo a una integración "natural" del software y de TIC en general al proceso de investigación cualitativa.

\section{Fases de la investigación a las que se asocia el uso de herramientas}

Consideramos fases del proceso investigativo aquellas que, más allá de los métodos, se realizan para la producción de conocimiento: Revisión de literatura, Definición de la muestra, Diseño de los instrumentos, Procesos de recolección de información, Sistematización y procesamiento de información, y Comunicación y divulgación de los resultados.

La mayor parte de la producción se agrupa principalmente en la fase de Sistematización y análisis de información y, en un segundo lugar, en la Recolección de información (Figura 6). Esto puede interpretarse como una consecuencia natural de los ítems anteriores, herramientas y enfoques temáticos, donde prima el CAQDAS. Sin embargo, es necesario señalar que la evolución y el refinamiento en las funcionalidades de este tipo de software incide en un uso más temprano en el proceso investigativo. Para ilustrar, se puede mencionar un proceso como el análisis de bibliografía para construir estados de la cuestión, una de las fases iniciales de la investigación.

Por otra parte, se ha ampliado la posibilidad de trabajar con formatos de datos diferentes: texto, imagen, audio, video e información de redes sociales 
como Twitter y Facebook, con lo cual no sólo se incrementan otros tipos de análisis (multimedial, de redes sociales) sino que se facilitan procesos como la recolección, el registro y la transcripción de información. Actualmente, software como NVivo (desde su versión 10) y ATLAS.ti 8 disponen de funciones que favorecen la integración con datos de tipo cuantitativo, con lo cual aumenta la variedad de fuentes de información y se gana en la riqueza y complejidad de los análisis.

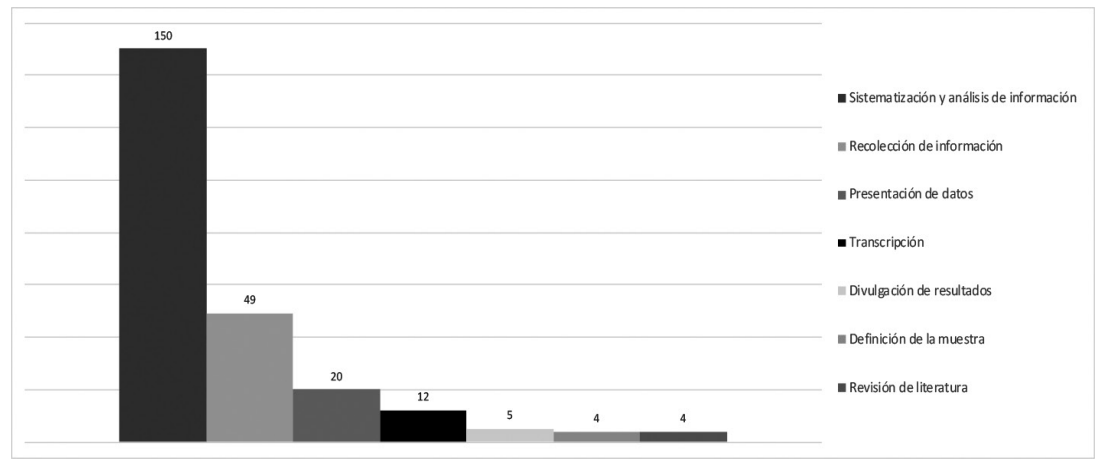

Figura 6. Número de artículos en los que se relacionan las fases de la investigación con el uso de herramientas TIC

DiSCUSIÓN: REPERTORIO DE USOS DE CAQDAS Y TIC DE ACUERDO CON LAS FASES DEL PROCESO INVESTIGATIVO

A continuación se presenta una triple relación entre dispositivos y software usados, formas de uso (repertorios) y fases del proceso investigativo en el que se implementan.

\section{Fase de la revisión de literatura}

Se puede hablar de dos usos de TIC relevantes que ya se han consolidado en las prácticas de algunos investigadores durante la realización de estados del arte: el primero es el uso generalizado de internet para acceder a la información científica, el segundo consiste en el empleo de software especializado para la gestión de referentes bibliográficos. Algunas de las razones y características destacadas por varios autores (De Bellis, 2009; Estalella y Ardévol, 2011; Jiménez-Contreras, 2000; Norman, 2010; Roy, Faulkner y Finlay, 2007; Suri y Clarke, 2009; Tarrats Pons, 2012) son las siguientes: 
- Los procesos de digitalización permitieron la ampliación del acceso a revistas científicas, tanto para consulta como para publicación. Además, se ampliaron las posibilidades de publicación en revistas y blogs de divulgación científica abierta que complementan toda la oferta de bases de datos comerciales (Paulus, Lester y Dempster, 2014; Paulus, Jackson y Davidson, 2017).

- Desarrollo de estrategias colaborativas y herramientas para compartir información bibliográfica entre investigadores, tales como los gestores bibliográficos y sus aplicaciones en la nube.

- Desarrollo de la bibliometría gracias a los "registros" de actividad en torno a la producción bibliográfica y la comunicación entre diferentes software por la compatibilidad de formatos y lenguajes.

\section{Fase de la definición de la muestra}

En esta fase autores como Alivernini, Lucidi y Manganelli (2008), Seawright y Gerring (2008), Snee (2008), Ison (2009), Colás Bravo y De Pablos Pons (2012) y Sigfridsson y Sheehan (2011) destacan usos de TIC específicos, como:

- Localización y contacto de casos "tipo" superando dificultades de lugar, tiempo y miedo a la participación.

- Estudio de comunidades virtuales.

- Inclusión de población con deficiencias de comunicación verbal.

- Selección de casos a través de métodos mixtos (apoyado en software estadístico).

\section{Fase del diseño de instrumentos y recolección de información}

Algunas de las investigaciones revisadas (Kosinski et al., 2015; Lijadi y Van Schalkwyk, 2015; López Cruz, Valdivia y Fernández Droguett, 2016; Marcu, 2015; Wilson, Kenny y Dickson-Swift, 2015) reportan cambios en el diseño de instrumentos y recolección de información que representan ventajas por las posibilidades de accesibilidad, ampliación, velocidad e incremento de las técnicas participativas de recolección. Varios de los aspectos que destacan son los siguientes:

- Ajuste a las nuevas formas de comunicación mediadas tecnológicamente como redes sociales, foros, chats, videoconferencias, tuits, entre otros, espacios para los cuales algunos software especializados ya 
desarrollaron herramientas de captura directa (Kosinski et al., 2015; Wishart y Thomas, 2018 y Kiyimba, Lester y O’Reilly, 2019).

- Disponibilidad de recursos de software abierto para el diseño de instrumentos (Survey Monkey, formularios de Google Drive) y la aplicación de encuestas, entre ellos las redes sociales, cada vez más usadas para este propósito (Evans y Mathur, 2005; Buchanan y Hvizdak, 2009 y Forgasz et al., 2018).

- Reducción de costos y tiempo, como en el caso de la comunicación asincrónica textual (como los foros de discusión) que no requieren transcripción (Álvarez, 2013).

- Accesibilidad a dispositivos móviles para el registro de información de audio y video, así como para el acceso de información multiplataforma, particularmente en casos de trabajos de etnografía virtual (Paulus et al., 2017 y Udovicich y Zanotti, 2017).

- Disponibilidad de datos en línea (Open Data Sources), la posibilidad de la preservación digital de datos y reutilización de los mismos (Mruck, 2005 y Corti y Fielding, 2016).

- Efecto desinhibidor en las entrevistas: para determinadas temáticas y poblaciones la entrevista en línea resulta más cómoda y permite una conversación más fluida (Procter, Carmichael y Laterza, 2008).

Dentro de este apartado incluimos los procesos de transcripción de información porque encontramos reportes muy interesantes al respecto que dimensionan la importancia no sólo de la recopilación de información sino de lo que se denomina normalización de datos para su posterior análisis:

- Transformación de la toma de nota manual: con la posibilidad de un registro fidedigno de las entrevistas mediante audio y video se releva al investigador de esta tarea para dedicarse a aspectos analíticos, contextuales y de orden pragmático (Farías y Montero, 2005; O’Brien et al., 2012; Opdenakker, 2006; Paulus y Lester, 2016).

- Surgimiento de modalidades de segmentación más ágiles directamente sobre audio y video que posibilitan el análisis de elementos no textuales del discurso, con lo cual la transcripción pasa a ser un proceso selectivo y analítico.

- Uso de software que permite el dictado por voz o el reconocimiento de voz desde una grabación que puede ser útil en investigaciones de casos únicos, tipo historias de vida o estudios biográficos, y como apoyo a las personas que realizan tareas de transcripción. Sin embargo, este tema está sujeto, hoy, a condiciones de entrenamiento del software con 
la voz y a la calidad de las grabaciones. Técnicamente es un tema que aún requiere más desarrollo; por ejemplo, en idiomas como el español resulta complejo debido, entre otras razones, a la riqueza lexical y a la variación de la entonación en las distintas regiones y países (Bhatt, De Roock y Adams, 2015).

\section{Fase de la sistematización y análisis de información}

Como se evidencia en la Figura 6, aquí se concentra la mayor parte de la literatura sobre el uso de CAQDAS y de TIC, que reportaron casos de uso y comparación de herramientas tipo CAQDAS (Angus, Rintel y Wiles, 2013; Evers et al., 2011; Karcher y Pagé, 2017; King, 2010; Konopásek 2007; Paulus et al., 2017; Sotiriadou, Brouwers y Le, 2014; Woods, Macklin y Lewis, 2016). Algunas de las características que más refiere la literatura en relación con las prestaciones y posibilidades que ofrece el software son:

- Integración de información en diversos formatos (audio, video, texto, imágenes, tablas de datos) facilitando la visualización de la información y la identificación de relaciones entre los datos: por ejemplo, el uso de redes semánticas, categorías, memos y comentarios que son la base de las primeras interpretaciones (Farías y Montero, 2005; Hatani, 2015; Markle, West y Rich, 2011; Parmeggiani, 2008, 2009; Woods y Dempster, 2011; Wright, 2016).

- Posibilidad de manejar grandes cantidades de información de manera organizada y colaborativa. La fusión de proyectos e intercambio de información entre investigadores para triangulación y validación de análisis (Rodik y Primorac, 2015).

- Uso de representaciones gráficas para exploración y presentación de información, dado el avance en las características del software cualitativo que ha permitido no sólo incorporar sino optimizar la visualización de los datos, que antes exigía un trabajo adicional por parte del investigador con otro tipo de software (Bencomo, Godino y Wilhelmi, 2004; Verd Pericás, 2005; Austin y Hickey, 2009; Parmeggiani, 2009; Pastor et al., 2012 y Angus, Rintel y Wiles, 2013).

- Integración de herramientas para búsquedas avanzadas en texto como conteo de palabras o búsquedas simultáneas de términos.

- Posibilidades de la codificación directa de audio y video (Silver y Patashnick, 2011). 


\section{Fase de divulgación y comunicación de resultados}

En lo referido a las formas de representación de los datos en investigación cualitativa no se encontró mucha literatura dedicada al tema en bases de datos académicas y científicas. Caso contrario sucede cuando se realiza esta búsqueda en internet, donde abundan blogs y sitios especializados que intentan poner al orden del día las posibilidades de visualización y representación de información, en estas fuentes se evidencia un cambio gradual a formas más dinámicas de visualización de los datos cualitativos, campo antes dominado por la graficación especializada de los datos cuantitativos. Las prácticas más destacadas por los autores son:

- Cambio en las formas de visualización de información cualitativa por el desarrollo del software para los diferentes procesos de la investigación. Acceso a recursos en línea que permiten la representación de información no estructurada (redes semánticas, georreferenciación, nubes de palabras, modelos gráficos, visualización compleja de datos) (Parmeggiani, 2009; Verd y Porcel, 2012; Fielding, 2012, 2018).

- Diversificación en las formas de presentación de informes de investigación que incluyen elementos multimediales (audio, video, fotografías) o presentación en formatos digitales dinámicos (hipertexto, hipermedia) (Lamarca Lapuente, 2006).

- Mayor facilidad para la presentación de datos "mixtos" a partir de la relación de datos cuantitativos y cualitativos dada la intercomunicabilidad y la funcionalidad del software de integrar ambos tipos de análisis en un mismo programa.

\section{Conclusiones}

Es importante resaltar las particularidades encontradas en la producción bibliográfica relacionada con los usos de software especializado tipo CAQDAS y de TIC en investigación cualitativa, tales como el centramiento en discusiones propias sobre el uso de las herramientas (Costa et al., 2018; Evers, 2018; Freitas et al., 2018) la abundancia de casos de uso, el predominio de las discusiones sobre los software tipo CAQDAS y los debates centrados en la pertinencia de su uso en los procesos investigativos cualitativos (Jackson, Paulus y Woolf, 2018; Paulus, Evers y Jong, 2018). Estas características no son tan marcadas en comparación con escenarios de uso de software especializado y de TIC en investigación como los estudios cuantitativos, lo que 
sugiere que nos encontramos en un proceso no culminado de comprensión y apropiación de estas herramientas en el proceso investigativo cualitativo.

La revisión permitió evidenciar una evolución en los procesos de apropiación de TIC y de software especializado que ha visto emerger prácticas investigativas, como uso más generalizado de CAQDAS para la revisión de literatura y el análisis documental (O’Neill, Booth y Lamb, 2018), incremento de información disponible que exige el desarrollo de nuevas habilidades para la búsqueda, selección y manejo de información bibliográfica frente a su crecimiento "exponencial" tanto en internet como en bases de datos especializadas; mayor diversidad y variación de la muestra, y funciones específicas para captura de información en línea proveniente de redes sociales o páginas web, que ya hoy incorporan los software especializados y que relevan al investigador de las tareas manuales de preparación de la información (Silver y Bulloch, 2016).

Esto plantea para las ciencias sociales dos posibles escenarios de cambio: el primero, el reto del uso de CAQDAS y de TIC por parte de una comunidad científica inmersa en el proceso de cambio tecnológico (Fielding, 2018), y el segundo, el creciente interés de algún sector de esta comunidad de hacer de este un campo de estudio privilegiado dentro de sus líneas de investigación.

\section{REFERENCIAS}

Alivernini, Fabio, Fabio Lucidi y Sara Manganelli. 2008. "Assessment of academic motivation: a mixed methods study". International Journal of Multiple Research Approaches 2 (1): 71-82.

https://doi.org/10.5172/mra.455.2.1.71

Álvarez, Gloria. 2013. "La interacción comunicativa en espacios virtuales de aprendizaje: un enfoque pragmático para el estudio de las discusiones mediadas por TIC”. Tesis de doctorado, Universidad de Salamanca, Facultad de Educación. http://gredos.usal.es/jspui/handle/10366/124045

Álvarez, Gloria, María Elena Giraldo y Clemencia Navarro. 2017. "Uso de TIC en investigación cualitativa: Discusión y tendencias en la literatura”. Katharsis 23: 218-235. https://doi.org/10.25057/25005731.873

Angus, Daniel, Sean Rintel y Janet Wiles. 2013. "Making sense of big text: a visual-first approach for analysing text data using Leximancer and Discursis". International Journal of Social Research Methodology 16 (3): 261-267. https://doi.org/10.1080/13645579.2013.774186

Arriazu Muñoz, Rubén. 2007. “¿Nuevos medios o nuevas formas de indagación?: una propuesta metodológica para la investigación social on-line a través del foro de discusión". Forum Qualitative Sozialforschung/Forum: Qualitative Social Research 8 (3). https://doi.org/10.17169/fqs-8.3.275 
Austin, Jon y Andrew Hickey. 2009. "Working visually in community identity ethnography”. International Journal of the Humanities 7 (4): 1-14. https://doi.org/10.18848/1447-9508/CGP/v07i04/42668

Bencomo, Delisa, Juan Godino y Miguel Wilhelmi. 2004. "Elaboración de redes ontosemióticas de configuraciones didácticas con ATLAS/ti”. Trabajo presentado en la primer Conferencia Internacional de Mapeo de Conceptos, Pamplona, España, 8-11 http://cmc.ihmc.us/papers/cmc2004-135.pdf

Bhatt, Ibrar, Roberto de Roock y Jonathon Adams. 2015. "Diving deep into digital literacy: emerging methods for research". Language and Education 29 (6): 477-492. https://doi.org/10.1080/09500782.2015.1041972

Bourdon, Sylvain. 2002. "The integration of qualitative data analysis software in research strategies: resistances and possibilities". Forum Qualitative Sozialforschung/Forum: Qualitative Social Research 3 (2). https://doi.org/10.17169/fqs-3.2.850

Buchanan, Elizabeth y Erin Hvizdak. 2009. "Online survey tools: ethical and methodological concerns of human research ethics committees". Journal of Empirical Research on Human Research Ethics 4 (2): 37-48. https://doi.org/10.1525/jer.2009.4.2.37

Chernobilsky, Lilia. 2006. "El uso de la computadora como auxiliar en el análisis de datos cualitativos", en Estrategias de Investigación Cualitativa, coordinado por Irene Vasilachis de Gialdino, 239-263. Barcelona: Gedisa.

Cisneros Puebla, César. 2003. "Análisis cualitativo asistido por computadora”. Sociologias 5 (9): 288-313. https://doi.org/10.1590/S1517-45222003000100010

Cisneros Puebla, César. 2011. "La computación cualitativa en la investigación en ciencias sociales”. La Sociología en sus Escenarios, 23: 1-27.

Cisneros Puebla, César, Daniel Figaredo, Robert Faux, Carlos Kölbl y Martín Packer. 2006. "About qualitative research epistemologies and peripheries". Forum Qualitative Sozialforschung/Forum: Qualitative Social Research 7 (4). http://www.qualitative-research.net/index.php/fqs/article/view/158

Cisneros Puebla, César y Judith Davidson. 2012. "Qualitative computing and qualitative research: addressing the challenges of technology and globalization". Forum Qualitative Sozialforschung/Forum: Qualitative Social Research 13 (2). https://doi.org/10.17169/fqs-13.2.1853

Cisneros Puebla, César, Robert Faux, Jo Moran-Ellis, Ercilia García-Álvarez y Jordi López-Sintas. 2009. "Introducción: cultivando los aspectos culturales al hacer investigación”. Forum Qualitative Sozialforschung/Forum: Qualitative Social Research 10 (2).

http://www.qualitative-research.net/index.php/fqs/article/view/1315

Colás Bravo, Pilar y Juan de Pablos Pons. 2012. "Aplicaciones de las tecnologías de la información y la comunicación en la investigación cualitativa”. Applications of information and communication technologies to qualitative research (251): 77-92.

Corti, Louise y Niguel Fielding. 2016. "Opportunities from the digital revolution: implications for researching, publishing, and consuming qualitative research”. Sage open 6 (4): 1-13. https://doi.org/10.1177/2158244016678912 
Costa, Antonio, Francislê De Souza, Antonio Moreira y Dayse De Souza. 2018. “webQDA 2.0 Versus webQDA 3.0: a comparative study about usability of qualitative data analysis software", en Developments and Advances in Intelligent Systems and Application, editado por Álvaro Rocha y Luis Reis, 229-240. Portugal: Springer International Publishing.

De Bellis, Nicola. 2009. Bibliometrics and citation analysis: from the science citation index to cybermetrics. United States of America: Scarecrow Press.

Estalella, Adolfo y Elisenda Ardévol. 2011. "E-research: desafíos y oportunidades para las ciencias sociales". Convergencia 18 (55): 87-111.

Evans, Joel y Anil Mathur. 2005. "The value of online surveys". Internet Research 15 (2): 195-219. https://doi.org/10.1108/10662240510590360

Evers, Jeanine. 2018. "Current issues in Qualitative Data Analysis Software (QDAS): a user and developer perspective”. The Qualitative Report 23 (13): 61-73.

Evers, Jeanine, Christina Silver, Katja Mruck y Bart Peeters. 2011. "Introduction to the KWALON experiment: discussions on qualitative data analysis software by developers and users". Forum Qualitative Sozialforschung/Forum: Qualitative Social Research 12 (1). https://doi.org/10.17169/fqs-12.1.1637

Farías, Levy y Maritza Montero. 2005. "De la transcripción y otros aspectos artesanales de la investigación cualitativa". International Journal of Qualitative Methods 4 (1): 53-68. https://doi.org/10.1177/160940690500400104

Fielding, Nigel. 2012. "The diverse worlds and research practices of qualitative software”. Forum Qualitative Sozialforschung/Forum: Qualitative Social Research 13 (2). https://doi.org/10.17169/fqs-13.2.1845

Fielding, Nigel. 2018. "New data and old dilemmas: changes and continuities in online social research”. Qualitative Inquiry 8 (25): 761-772. https://doi.org/10.1177/1077800418809130

Fielding, Nigel, Raymon Lee y Wilma Mangabeira. 2004. "Computers and qualitative research adoption, use, and representation". Social Science Computer Review 22 (2): 167-178.

https://doi.org/10.1177/0894439303262622

Fielding, Nigel y Raymon Lee. 1996. "Qualitative data analysis: representations of a technology: a comment on Coffey, Holbrook and Atkinson”. Sociological Research Online 1 (4): 1-6. https://doi.org/10.5153/sro.1326

Fielding, Roy, James Gettys, Jeff Mogul, Henrik Frystyk, Larry Masinter, Paul Leach y Lee Berners. 1999. Hypertext transfer protocol-bttp/1.1. Rfc 2616, June. http://www.hjp.at/doc/rfc/rfc2616.html

Forgasz, Helen, Hazel Tan, Gilah Leder y Amber McLeod. 2018. "Enhancing survey participation: Facebook advertisements for recruitment in educational research". International Journal of Research \& Method in Education 41 (3): 257-270. https://doi.org/10.1080/1743727x.2017.1295939

Freitas, Fabio, Jaime Ribeiro, Catarina Brandão, Francislê De Souza, Antonio Costa y Luis Reis. 2018. "In case of doubt see the manual: a comparative analysis of (self)learning packages qualitative research software", en Computer Supported Qualitative Research, editado por Antonio Costa, Francislê De Souza, Luis Reis y Antonio Moreira, 176-192. Portugal: Springer International Publishing. 
Gibbs, Graham. 2013. "Using software in qualitative analysis”, en The Sage Handbook of Qualitative Data Analysis, editado por Uwe Flick, 277-296. Inglaterra: Sage Publications.

Hatani, Faith. 2015. "Analyzing high-profile panel discussion on global health: an exploration with MAXQDA”. Forum Qualitative Sozialforschung/Forum: Qualitative Social Research (16).

http://www.qualitative-research.net/index.php/fqs/article/view/2208

Hine, Chirstine. 2000. Virtual Ethnography. Inglaterra: Sage Publications. https://books.google.com.co/books?id=X5w1P2_iMNYC\&printsec=frontcover $\& \mathrm{hl}=\mathrm{es} \&$ source $=\mathrm{gbs} \_\mathrm{ge} \_$summary_r $\& \mathrm{cad}=0 \# \mathrm{v}=$ onepage $\& \mathrm{q} \& \mathrm{f}=\mathrm{false}$

Ison, Nicole. 2009. "Having their say: email interviews for research data collection with people who have verbal communication impairment”. International Journal of Social Research Methodology 12 (2): 161-172. https://doi.org/10.1080/13645570902752365

Jackson, Kristi, Trena Paulus y Nicholas Woolf. 2018. “The walking dead genealogy: unsubstantiated criticisms of Qualitative Data Analysis Software (QDAS) and the failure to put them to rest". The Qualitative Report 23(13): 74-91.

Jiménez-Contreras, Evaristo. 2000. "Los métodos bibliométricos: aplicaciones y estado de la cuestión”. Trabajo presentado en I Congreso Universitario de Ciencias de la Documentación. Teoría, Historia y Metodología de la Documentación en España, Madrid, 14-17 de noviembre. http://eprints.rclis.org/14039/

Karcher, Sebastian y Chistiane Pagé. 2017. "Workshop report: CAQDAS projects and digital repositories' best practices”. D-Lib Magazine 23(3/4). https://doi.org/10.1045/march2017-karcher

King, Andrew. 2010. "Membership matters: applying Membership Categorization Analysis (MCA) to qualitative data using Computer-Assisted Qualitative Data Analysis (CAQDAS) software". International Journal of Social Research Methodo$\log y 13$ (1): 1-16.

https://doi.org/10.1080/13645570802576575

Kiyimba, Nikki, Jessica Lester y Michelle O’Reilly. 2019. "Utilising computer-mediated communication and the internet in health research", en Using Naturally Occurring Data in Qualitative Health Research: A practical guide, editado por Nikki Kiyimba, Jessica Lester y Michelle O'Reilly, 179-203. Cham: Springer International Publishing. https://doi.org/10.1007/978-3-319-94839-3_8

Konopásek, Zdeněk. 2007. "Making thinking visible with ATLAS.Ti: computer assisted qualitative analysis as textual practices”. Supplement, Historical Social Research 19: 276-298.

Kosinski, Michal, Sandra Matz, Samuel Gosling, Vesselin Popov y David Stillwell. 2015. "Facebook as a research tool for the social sciences: opportunities, challenges, ethical considerations, and practical guidelines". American Psychologist 70 (6): 543-556.

https://doi.org/10.1037/a0039210

Lamarca Lapuente, María. 2006. "Hipertexto: el nuevo concepto de documento en la cultura de la imagen". Tesis de doctorado, Universidad Complutense de Madrid, Facultad de Ciencias de la Información.

http://www.hipertexto.info 
Lijadi, Anastasia y Gertina Van Schalkwyk. 2015. "Online Facebook focus group research of hard-to-reach participants”. International Journal of Qualitative Methods 14 (5): 1-9. https://doi.org/10.1177/1609406915621383

López Cruz, Mauricio, Andrea Valdivia y Roberto Fernández Droguett. 2016. "Producciones narrativo-visuales y voz de los y las estudiantes: indagación sobre los significados de participación en niños, niñas y jóvenes en escuelas municipales en Chile”. Forum Qualitative Sozialforschung/Forum: Qualitative Social Research 17 (1).

MacMillan, Katie y Thomas Koenig. 2004. "The Wow factor preconceptions and expectations for data analysis software in qualitative research". Social Science Computer Review 22 (2): 179-186. https://doi.org/10.1177/0894439303262625

Marcu, Oana. 2015. "Utilizando métodos biográficos, participativos y visuales con jóvenes romaníes”. Forum Qualitative Sozialforschung/Forum: Qualitative Social Research 17 (1). http://www.qualitative-research.net/index.php/fqs/article/view/2270

Markle, Thomas, Richard West y Peter Rich. 2011. "Beyond transcription: technology, change, and refinement of method”. Forum Qualitative Sozialforschung/ Forum: Qualitative Social Research 12 (3). https://doi.org/10.17169/fqs-12.3.1564

Mruck, Katja. 2005. "Providing (online) resources and services for qualitative researchers: challenges and potentials". Forum Qualitative Sozialforschung/Forum: Qualitative Social Research 6 (2). https://doi.org/10.17169/fqs-6.2.461

Norman, Farley. 2010. “Bibliographic Management 2.0”. eLucidate 7 (2): 3-7.

O’Brien, Mark, Tunde Varga-Atkins, Muriah Umoquit y Peggy Tso. 2012. "Culturalhistorical activity theory and 'the visual' in research: exploring the ontological consequences of the use of visual methods". International Journal of Research and Method in Education 35 (3): 251-268. https://doi.org/10.1080/1743727X.2012.717433

O’Neill, Maureen, Sarah Booth y Janeen Lamb. 2018. “Using NVivoTM for literature reviews: the eight step pedagogy (N7+1)”. The Qualitative Report 23 (13): 21-39.

Opdenakker, Raymond. 2006. "Advantages and disadvantages of four interview techniques in qualitative research". Forum Qualitative Sozialforschung/Forum: Qualitative Social Research 7 (4).

http://www.qualitative-research.net/index.php/fqs/article/view/175

Parmeggiani, Paolo. 2008. "Teaching different research methods through the use of video analysis software for media students: a case study". International Journal of Multiple Research Approaches 2 (1): 94-104. https://doi.org/10.5172/mra.455.2.1.94

Parmeggiani, Paolo. 2009. "Going digital: using new technologies in visual sociology”. Visual Studies 24 (1): 71-81. https://doi.org/10.1080/14725860902732991 
Pastor, Liliana, Ricardo Chrobak, Erika Chrobak, María Ponzoni, Patricia Barraza y Gabriela Rodríguez. 2012. "Investigación cualitativa: mapas conceptuales generados a partir del software ATLAS.ti y el aprendizaje significativo de los investigadores”. Trabajo presentado en Concept Maps: theory, methodology, technology. Proceedings of the Fifth International Conference on Concept Mapping, Valleta, Malta, 5-7 de septiembre. http://cmc.ihmc.us/cmc2012papers/cmc2012-p157.pdf

Paulus, Trena, Jeanine C. Evers y Franciska de Jong. 2018. "Reflecting on the future of QDA software: Special issue of The Qualitative Report". The Qualitative Report 23 (13): 1-5.

https://nsuworks.nova.edu/tqr/vol23/iss13/1/

Paulus, Trena, Jessica Lester y Paul Dempster. 2014. Digital tools for qualitative research. Inglaterra: Sage.

Paulus, Trena, Kristi Jackson y Judith Davidson. 2017. "Digital tools for qualitative research: disruptions and entanglements”. Qualitative Inquiry 23 (10): 751-756. https://doi.org/10.1177/1077800417731080

Paulus, Trena y Jessica Lester. 2016. “ ATLAS.ti for conversation and discourse analysis studies”. International Journal of Social Research Methodology 19 (4): 405-428. https://doi.org/10.1080/13645579.2015.1021949

Paulus, Trena, Megan Woods, David Atkins y Rob Macklin. 2017. “The discourse of QDAS: reporting practices of ATLAS.ti and NVivo users with implications for best practices". International Journal of Social Research Methodology 20 (1): 35-47. https://doi.org/10.1080/13645579.2015.1102454

Peter, Ian. 2010. “The history of email”. Nethistory. http://www.nethistory.info/History\%20of\%20the\%20Internet/email.html

Procter, Richard, Patrick Carmichael y Vito Laterza. 2008. "Co-interpretation of usage data: a mixed methods approach to evaluation of online environments". International Journal of Multiple Research Approaches 2 (1): 44-56. https://doi.org/10.5172/mra.455.2.1.44

Roberts, Kathryn y Richard Wilson. 2002. "ICT and the research process: issues around the compatibility of technology with qualitative data analysis". Forum Qualitative Sozialforschung/Forum: Qualitative Social Research 3 (2). https://doi.org/10.17169/fqs-3.2.862

Rodik, Petra y Jaka Primorac. 2015. "To use or not to use: Computer-Assisted Qualitative Data Analysis Software usage among early-career sociologists in Croatia”. Forum Qualitative Sozialforschung/Forum: Qualitative Social Research 16 (1). https://doi.org/10.17169/fqs-16.1.2221

Roy, Stephannie, Guy Faulkner y Sara Finlay. 2007. "Hard or soft searching? Electronic database versus hand searching in media research". Forum Qualitative Sozialforschung/Forum: Qualitative Social Research 8 (3). http://www.qualitative-research.net/index.php/fqs/article/view/289

Seawright, Jason y John Gerring. 2008. "Case selection techniques in case study research a menu of qualitative and quantitative options". Political Research Quarterly 61 (2): 294-308.

https://doi.org/10.1177/1065912907313077 
Sigfridsson, Anders y Anne Sheehan. 2011. "On qualitative methodologies and dispersed communities: reflections on the process of investigating an open source community". Information and Software Technology 53 (9): 981-993.

https://doi.org/10.1016/j.infsof.2011.01.012

Silver, Christina y Sarah Bulloch. 2016. "CAQDAS at a crossroads: affordances of technology in an online environment", en The SAGE Handbook of Online Research Methods, editado por Nigel Fielding, Raymond Lee y Grant Blank. Inglaterra: Sage. https://doi.org/10.4135/9781473957992.n27

Silver, Christina y Jennifer Patashnick. 2011. "Finding fidelity: advancing audiovisual analysis using software". Forum Qualitative Sozialforschung/Forum: Qualitative Social Research 12 (1). https://doi.org/10.17169/fqs-12.1.1629

Sin, Chih. 2008. "Teamwork involving Qualitative Data Analysis Software striking a balance between research ideals and pragmatics". Social Science Computer Review 26 (3): 350-358.

https://doi.org/10.1177/0894439306289033

Snee, Helene. 2008. Web 2.0 as a social science research tool. Inglaterra: British Library.

Sotiriadou, Popi, Jessie Brouwers y Tuan-Anh Le. 2014. "Choosing a qualitative data analysis tool: a comparison of NVivo and Leximancer”. Annals of Leisure Research 17 (2): 218-234. https://doi.org/10.1080/11745398.2014.902292

Suri, Harsh y David Clarke. 2009. "Advancements in research synthesis methods: from a methodologically inclusive perspective”. Review of Educational Research 79 (1): 395-430.

https://doi.org/10.3102/0034654308326349

Tarrats Pons, Elisenda. 2012. "Sitkis: una herramienta bibliométrica para el desarrollo del estado de la cuestión". BiD. Textos universitaris de biblioteconomía $i$ documentació 28 (junio)

https://doi.org/10.1344/105.000001831

Trejo Teruel, Elvis. 2011. "Soporte informático para la investigación cualitativa: caso de los programas ATLAS.ti y NVIVO”. Población y Desarrollo-Argonautas y Caminantes (4): 86-109.

https://doi.org/10.5377/pdac.v4i0.309

Udovicich, Mariana y Agustín Zanotti. 2017. "Análisis etnográfico multi-plataforma. Inmersiones en internet y desafíos de campo”. Forum Qualitative Sozialforschung/Forum: Qualitative Social Research 18 (3). https://doi.org/10.17169/fqs-18.3.2645

Valles, Miguel. 2001. "Ventajas y desafíos del uso de programas informáticos (eg ATLAS.ti y Maxqda) en el análisis cualitativo. Una reflexión metodológica desde la grounded theory y el contexto de la investigación social española". Trabajo presentado en el I Seminario sobre Investigación Avanzada Cualitativa Asistida por Ordenador, Granada, España, 22-23 de noviembre.

http://centrodeestudiosandaluces.es/biblio/imagendoc/00000001_00000500/0 0000125/00000125_090h0101.pdf

Valles, Miguel. 2005. "Metodología y tecnología cualitativas: actualización de un debate, desde la mirada más atenta en la obra de Barney G. Glaser”. Empiria: revista de metodología de ciencias sociales (9): 145-170. https://doi.org/10.5944/empiria.9.2005.1007 
Verd, Joan y Sergio Porcel. 2012. "An application of qualitative Geographic Information Systems (GIS) in the field of urban sociology using ATLAS.ti: uses and reflections”. Forum Qualitative Sozialforschung/Forum: Qualitative Social Research 13 (2). https://doi.org/10.17169/fqs-13.2.1847

Verd Pericás, Miguel. 2005. "El uso de la teoría de redes sociales en la representación y análisis de textos. De las redes semánticas al análisis de redes textuales”. Empiria: revista de metodología de ciencias sociales (10): 129-150. https://doi.org/10.5944/empiria.10.2005.1046

Wilson, Elena, Amanda Kenny y Virginia Dickson-Swift. 2015. "Using blogs as a qualitative health research tool: a scoping review”. International Journal of Qualitative Methods 14 (5): 1-12. https://doi.org/10.1177/1609406915618049

Wishart, Jocelyn y Michael Thomas. 2018. E-research in educational contexts: the roles of technologies, ethics and social media. Inglaterra: Routledge.

Woods, David y Paul Dempster. 2011. "Tales from the bleeding edge: the qualitative analysis of complex video data using transana". Forum Qualitative Sozialforschung/Forum: Qualitative Social Research 12 (1). https://doi.org/10.17169/fqs-12.1.1516

Woods, Megan, Rob Macklin y Gemma Lewis. 2016. "Researcher reflexivity: exploring the impacts of CAQDAS use". International Journal of Social Research Methodology 19 (4): 385-403. https://doi.org/10.1080/13645579.2015.1023964

Wright, Steven. 2016. "Exploring actor-network theory and CAQDAS: provisional principles and practices for coding, connecting and describing data using ATLAS.ti". https://doi.org/10.14279/depositonce-5153

Para citar este texto:

Giraldo Ramírez, María Elena, Gloria M. Álvarez Cadavid y Clemencia del Pilar Navarro Plazas. 2020. "Usos de TIC y software especializado en la investigación cualitativa. Un panorama”. Investigación Bibliotecológica: archivonomía, bibliotecología e información 34 (84): 33-57. http://dx.doi.org/10.22201/iibi.24488321xe.2020.84.58153 